\title{
The Apparent Stratification at the Top of Earth's Liquid Core
}

3 Jon Mound ${ }^{* 1}$, Chris Davies ${ }^{1}$, Sebastian $\operatorname{Rost}^{1} \&$ Jon Aurnou $^{2}$

${ }_{4}{ }^{1}$ School of Earth and Environment, University of Leeds, Leeds LS2 9JT, UK

$5{ }^{2}$ Department of Earth and Space Sciences, University of California, Los Angeles, California 6 90095-1567, USA.

7 Earth's magnetic field is generated by turbulent motion in its fluid outer core. Although the bulk of the outer core is vigorously convecting and well-mixed, some seismic, geomagnetic, and geodynamic evidence suggests that a global stably stratified layer exists at the top of Earth's core. Such a layer would strongly influence thermal, chemical, and momentum exchange across the core-mantle boundary (CMB) and thus have significant implications for the dynamics and evolution of the core. Here we argue that the relevant scenario is not global but regional stratification arising solely from the lateral variations in CMB heat flux. Based on our extensive suite of numerical simulations we expect that these regional inversion layers extend 100s of kilometres into the core under anomalously hot regions of the lowermost mantle. Although the majority of the outermost core remains actively convecting, sufficiently large and strong regional inversion layers produce a 1D temperature profile that mimics a globally stratified layer below the CMB, an apparent thermal stratification despite the average heat flux across the CMB being strongly superadiabatic. 
are controversial. Seismic wave speeds at the top of the core ${ }^{1,2}$ have been matched to a compositional model ${ }^{3}$ and interpreted as the signature of a global layer that is both thick $(\sim 300 \mathrm{~km})$ and strongly stratified (Brunt-Väisälä periods of 1.63-3.43 hr). Geomagnetic oscillations have been interpreted as the signature of MAC (Magnetic, Archimedes, and Coriolis) waves within a stratified layer $\sim 140 \mathrm{~km}$ thick with a maximum Brunt-Väisälä frequency that is roughly diurnal ${ }^{4,5}$; although this explanation is not unique ${ }^{6}$. Core flow models constructed from geomagnetic secular variation have been used to argue both for and against radial motion near the top of the core ${ }^{7-10}$ and some seismic studies ${ }^{11,12}$ have found that the structure of the outermost core does not require global stratification. Core stratification would also influence the long term thermal evolution of the $\operatorname{core}^{13}$; support a range of wave dynamics not found in a fully convecting $\operatorname{core}^{14}$; and, by suppressing radial motion near the $\mathrm{CMB}$, alter the long-term structure of the external planetary magnetic field $^{15,16}$.

Vigorous rotationally influenced flows within the electrically conductive liquid iron outer core are essential for the continued regeneration of the Earth's magnetic field through the magnetohydrodynamic geodynamo process. There is little doubt that the bulk of Earth's liquid core is undergoing turbulent convection and the horizontal temperature fluctuations within the adiabatically well-mixed fluid are expected to be very small $\left(\mathrm{o}\left\{10^{-3} \mathrm{~K}\right\}\right)^{17}$. Comparatively large radial variations in core properties can exist near the boundaries of the liquid core if some mechanism enables the generation or accumulation of fluid with a stable density stratification.

Three principle mechanisms have been invoked to explain a global non-adiabatic structure 
at the top of the core. The first supposes that the core has slowly cooled to a point where the heat flux, $q$, has fallen below the adiabatic heat flux, $q_{\mathrm{a}}$, across the $\mathrm{CMB}^{13}$. This scenario produces a wide range of thickness estimates ${ }^{18}$ that rely on the poorly-known CMB heat flow and muchdebated core conductivity ${ }^{19}$. The second mechanism invokes chemical diffusion, either along the core pressure gradient ${ }^{20}$ or across the $\mathrm{CMB}$ from the mantle ${ }^{21}$, which enriches the top of the core in light elements. The third possibility is emplacement of a light layer during core formation ${ }^{22}$, which must then avoid disruption throughout the lifetime of the Earth, or by the moon-forming $\operatorname{impact}^{23}$.

The top of the core will also be strongly influenced by thermal heterogeneity in the lowermost mantle, which is much stronger than in the core $\left(\mathrm{o}\left\{10^{2} \mathrm{~K}\right\}\right)$ and evolves much more slowly, such that the mantle imposes a laterally varying pattern of heat flux across the core-mantle boundary $(\mathrm{CMB})^{24}$. Estimates of the lateral variations in CMB heat flux ${ }^{25-27}$ are sufficiently large that significant regional variations in core dynamics are expected ${ }^{16,28-31}$. Previous models ${ }^{16,32-34}$ have considered the interaction between $\mathrm{CMB}$ heterogeneity and stratification at the top of the core and the extent to which such heterogeneity can drive flows that penetrate and possibly disrupt a global stratified layer ${ }^{24,35}$. Rather than viewing heterogeneous CMB heat flux as a factor acting in opposition to some mechanism of global stratification we instead argue that it is the source of an apparent global stratification at the top of the core.

By utilising an extensive suite of nonmagnetic rotating convection simulations we have been able to systematically access the strongly nonlinear, rotationally constrained, turbulent flow regime 
most relevant to the Earth's core. Within this regime we find that the bulk of the core, including much of its surface, remains actively convecting due to a strong net superadiabatic heat flow across the CMB. Sufficiently warm regions in the lowermost mantle may locally reduce $q$ below $q_{\mathrm{a}}$ allowing regional accumulations of hot fluid at the top of the core within which the radial temperature gradient $(\partial T / \partial r)$ is locally positive. The spatial extent and buoyancy anomaly of these convectively-stable sub-CMB lenses of fluid, which we call regional inversion layers, are primarily set by the long-wavelength high-amplitude variations in CMB heat flux imposed on the core by the mantle. Large and strong regional inversion layers can dominate the spherically averaged temperature profile resulting in an apparent thermal stratification near the top of the core. There is no doubt that the fundamental physical mechanism that underpins our scenario, namely large lateral variations in CMB heat flux, exists within the $\operatorname{Earth}^{25,26,36}$; the only question is how significant its influence might be. Thick regional inversion layers are ubiquitous in our simulations and, we argue, should be expected in the Earth's core.

\section{Modelling of Regional Inversion Layers}

We investigate regional inversion layers in the core using a suite of numerical simulations of nonmagnetic rotating convection that includes two patterns (see supplementary figure 1) and two amplitudes of CMB heat flux heterogeneity (see methods and our previous work ${ }^{31}$ ). The amplitude of CMB heat flux heterogeneity in our numerical model is described by $q^{\star}=\frac{q_{\max }-q_{\min }}{q_{\mathrm{ave}}}$, where $q_{\mathrm{max}}, q_{\mathrm{min}}$, and $q_{\text {ave }}$ are the maximum, minimum and horizontally averaged heat fluxes through the outer boundary, respectively. In this study we consider strong lateral variations in CMB heat flux with $q^{\star}=\{2.3,5.0\}$. One pattern of CMB heat flux heterogeneity is derived from seismic 
tomography ${ }^{36}$. Laterally and radially extensive regions of low seismic velocity in the lowermost mantle, termed Large Low Velocity Provinces (LLVPs), have been observed and are hypothesised to arise from either thermal or thermochemical mechanisms ${ }^{37}$. In either case, these regions are expected to be anomalously warm and impose a reduced CMB heat flux on the core beneath Africa and the Pacific. The second is a hemispheric pattern that could represent the configuration of mantle flow during times of super-continent formation. For our chosen hemispheric pattern, $q_{\min }$ is located under Null Island $\left(0^{\circ} \mathrm{N}, 0^{\circ} \mathrm{E}\right)$.

Numerical models of core convection can be characterised by three control parameters: the Prandtl number $(P r)$, which is the ratio of the fluid's viscous and thermal diffusivities; and the Rayleigh number $(\widetilde{R a})$ and Ekman number $(E)$, which primarily reflect balances between rotational, viscous and buoyancy forces. Consideration based on the force balance between inertia, viscosity, and rotation suggests that the dynamic regime be characterised using the Reynolds number, $R e=U L / \nu$, and Rossby number, $R o=U / 2 \Omega L=R e E$, where $U$ and $L$ are the characteristic velocity and length scale of the flow, respectively, and $\nu$ is the momentum diffusivity. Our simulations consider higher $\widetilde{R a}$ and lower $E$ than previous models that incorporate CMB heat flux heterogeneity ${ }^{16,29,30}$. In particular, values of $E<10^{-4}$ allow us to access the regime of rapidlyrotating convection ${ }^{31,38}$. We also restrict our attention to simulations for which $\widetilde{R a}$ is at least ten times greater than the critical Rayleigh number for the onset of convection $\left(\widetilde{R a}_{\mathrm{c}}\right)$ to ensure that we have left the weakly non-linear regime near the onset of convection. Crucially our choice of control parameters results in the fluid flow in our simulations being both turbulent (large $R e$ ) and strongly influenced by rotation (small Ro) as in Earth's core (Table 1). 
In all of our simulations we find that convectively-stable regions of thermal inversion $(\mathrm{d} T / \mathrm{d} r>$ 0) can be maintained over large lateral and radial extents, although the bulk of the core remains strongly convecting and hence well mixed on short length scales (figures 1,2). The size of the regional inversion layers are associated with the long wavelengths of the imposed boundary heterogeneity rather than the small wavelengths of the convecting core (figure 1, supplementary figure 2, supplementary movies 1 and 2). Indeed the small scales of the convective fluctuations associated with strongly supercritical convection inhibit their ability to disrupt the large regions of thermal inversion ${ }^{39}$. Previous studies at low $\widetilde{R a}$ did not find the stratification signal ${ }^{29}$, perhaps because the potentially stable regions were disrupted by the large scale convective patterns that arise close to onset.

Regional inversion layers form underneath areas where the local CMB heat flux is sufficiently low to suppress convection near the top of the core. For our patterns of heterogeneity (supplementary figure 1), the CMB heat flux minima occur at or near the equator and thus the geographic profiles considered in figures 2 and 3 focus there. Even in regions where the CMB heat flux remains superadiabatic an inversion layer can exist a few hundred kilometres below the CMB as azimuthal flow sweeps hot material horizontally; see, for example, the volume of fluid with $\mathrm{d} T / \mathrm{d} r>0$ that extends west from the Pacific in figure 1. Enhanced CMB heat flux, relative to that underneath the LLVP, cools this westward extension of the Pacific inversion layer from above until the fluid becomes locally unstable with respect to thermal convection and mixes back into the bulk (see supplementary movie 1). 
The strength of the thermal inversion is characterised by the maximum Brunt-Väisälä frequency $(N)$, which we normalise relative to $2 \Omega$ (twice the planetary rotation rate). Scaling analysis (see methods) shows that the strength of the inversion should vary as

$$
\left.\frac{N}{2 \Omega}\right|_{\max } \approx\left(\frac{1}{r_{\mathrm{o}}^{\star}}\right) \sqrt{\frac{\widetilde{R a} E}{\operatorname{Pr}}\left(\frac{q^{\star}-2}{2}\right)},
$$

where $r_{\mathrm{o}}^{\star}$ is the dimensionless CMB radius. Extrapolation to the Earth must therefore account for both the increase in $\widetilde{R a}$ and the decrease in $E$ relative to numerical simulations (table 1). Stronger boundary heterogeneity (larger $q^{\star}$ ) implies more anomalous $\mathrm{d} T / \mathrm{d} r$ at the CMB and we expect $N^{2}$ to increase in proportion to $q^{\star}$.

The value of $q^{\star}$ can be estimated from first-principles calculations of thermal conductivity coupled to seismic tomographic models ${ }^{26}$ that suggest heat flux across the CMB ranges from roughly $0-140 \mathrm{~mW} / \mathrm{m}^{2}$. Much of the net radial heat flow within the core occurs due to conduction along the adiabatic temperature gradient ${ }^{19}$; this contribution needs to be removed when considering the relation between our Boussinesq model and the Earth. The super-adiabatic heat flow across the CMB has been estimated as 0.6 TW based on a theoretical scaling between inertial and buoyancy forces in rotating convection ${ }^{17}$. These values suggest $q^{\star}$ for the Earth may be as large as $\sim 35$, in which case $N / 2 \Omega \approx 2$ is predicted for the Earth for reasonable estimates of other physical parameters (supplemental table 1).

No theoretical scaling exists for the thickness of the regional inversion layers; they are not simple boundary layers, which would thin both as $\widetilde{R a}$ is increased and as $E$ is decreased towards Earth-like values. Instead we find a competition between thinner layers as the Ekman number is 
reduced but generally thicker layers as the Rayleigh number is increased for a given choice of $q^{\star}$ and CMB heat-flux pattern (figure 2 and supplemental figures 2 and 3 ).

Regional inversion layers that are both thick (several hundred kilometres) and strong $(N / 2 \Omega \approx$ $\left.\left\{10^{-2}-10^{0}\right\}\right)$ are ubiquitous in our models. The derived expression for Brunt-Väisälä frequency suggests that regional thermal stratification should be expected at low $E$, provided $\widetilde{R a}$ or $q^{\star}$ are sufficiently large. If the regional inversion layers are sufficiently large and strong, the horizontallyaveraged temperature gradient near the top of the core can become positive (figures 2,3), an apparent global stratification despite the average heat flux across the CMB being strongly superadiabatic. This apparent global stratification signal becomes stronger as $\widetilde{R a}$ is increased and the bulk of the core becomes more isothermal, thereby causing the horizontally averaged temperature gradient near the top of the core to be increasingly dominated by the large gradients that exist in the regional inversion layers.

\section{Discussion}

Previous dynamical modelling ${ }^{16,24,32-35}$ has focussed on interactions between heterogeneous boundary conditions and global stratified layers at the top of the core, motivated by stratification origins asuming uniform compositional enrichment ${ }^{20-22}$ or net subadiabatic CMB heat flux ${ }^{13,18}$. In contrast, our simulations do not impose a net stratification as they are all strongly supercritical and have a completely well-mixed fluid core in the absence of CMB heterogeneity. However, thermal variations in Earth's lowermost mantle are sufficiently strong that large areas of the CMB are expected to have have a subadiabatic heat flux ${ }^{25,26,36}$. Such areas locally inhibit convection in the 
outermost core, although the bulk of the core remains vigorously convecting and radial motion is not completely suppressed within the regional inversion layers (figure 4). Apparent global stratification arises as a consequence of CMB heterogeneity when the regional inversion layers control the sign of the global average radial temperature gradient, which is particularly likely at the high Rayleigh number conditions relevant to the Earth. The strength and extent of these regions is set by the boundary heterogeneity, which is faithfully represented in our simulations; therefore, we argue that broad and thick regional inversion layers should be expected in the Earth.

For the present day Earth, CMB heat flux is particularly low under the African and Pacific LLVPs and thus regional inversion layers are expected to be most prominent in these equatorial regions. If the pattern of mantle convection in the geological past had an approximately hemispheric pattern $^{40}$, then the regional inversion layers at that time would be expected to have a hemispheric pattern (see supplementary figures 2 and 3). The distribution of regional inversion layers in the past might be reflected in other large scale core processes that have been linked to mantle control, such as the structure and reversal rate of the magnetic field ${ }^{27,41,42}$ and the, possibly asymmetric, growth of the inner core $27,43,44$.

Unlike our Boussinesq numerical model, the anomalous regions in Earth's core need not have a strictly positive thermal gradient, they need only have a subadiabatic gradient to be dynamically distinct from the bulk of the core. The temperature difference between the top of actively convecting regions and the regional inversion layers depends on the layer thickness, $q^{\star}$ and the net superadiabatic heat flow across the CMB. Assuming purely thermal convection a simple theoreti- 
cal analysis suggests that the boundary-forced temperature variations can be orders of magnitude larger than those associated with the free convection (see methods, supplementary figure 5); however, temperature is believed to have only a moderate impact on seismic velocity in the core ${ }^{45}$. Chemical variations are expected to have a larger impact but the resultant seismic velocity relies on uncertain quantities such as the core's bulk composition, the nature of any chemical variation, and the impact of different chemical species on bulk modulus and density ${ }^{3,46,47}$. Our simulations are designed to elucidate the fluid dynamics of regional inversion layer formation due to CMB heat flux heterogeneity and provide a basis for future models incorporating processes such as barodiffusion, chemical exchange across the $\mathrm{CMB}$, and primordial stratification that have been hypothesised to influence the composition of the outermost core.

Although radial motion would be inhibited within a strongly stratified global layer, the regional inversion layers in our simulations are dynamically connected to the rest of the core; thus radial velocity is not completely suppressed within them (figure 4). The lateral variations in CMB heat flux drive thermal winds that sweep hot material out from under the locally stable regions of low CMB heat flux, enabling it to cool and mix back into the vigorously convecting bulk. This results in broad weak upwellings through the regional inversion layers in our simulations. In the Earth, such flows are also expected but may be modulated by other factors, such as magnetic field effects $^{24}$. Such boundary-driven flows have been used in previous dynamo studies ${ }^{48-50}$ to explain long-term non-axisymmetric features of the geomagnetic field.

Regional inversion layers may influence observable geomagnetic variation as both the wave 
dynamics and fluid flow (figure 4) in these regions would have a different character to that in the bulk of the core. Hemispheric patterns in geomagnetic secular variation ${ }^{51}$ may suggest that only one dominant regional inversion layer is present. In our model the Large Low Velocity Provinces are associated with low CMB heat flux and thus regional inversion layers; however, the latitudinal and longitudinal extents of the two LLVPs are quite different, which could result in differing influences on core thermal structure and hence geomagnetic variation. A hemispheric difference could also arise due to differences in temperature between the Pacific and African LLVPs, which might reflect differing balances between thermal and chemical contributions to these LLVPs origins. We find that the CMB heat flux reduction predicted by our chosen tomographic model is greater under the Pacific LLVP and this regional inversion layer tends to form more readily and be more extensive than the African. A hemispheric difference at the top of the core might therefore indicate that the average heat flux across the CMB is sufficiently hight to prevent regional inversion under Africa but not the Pacific. Uneven growth of the inner core ${ }^{52,53}$ might also produce large length scale differences in core dynamics that could influence hemispheric structures and dynamics at the top of the core $\mathrm{ca}^{49,50,54}$.

Without sufficient geographic coverage or understanding of how the path-integrated delay of $S m K S$ phases are influenced by regional inversion layers (for example, from 3D wave-propagation models), studies of average structure might well mistake extensive regional inversion layers for global stratification. The geometry and strength of regional inversion layers in the core depends on the pattern and amplitude of the imposed heat flux heterogeneity, which is set by the distributions of both temperature and thermal conductivity in the lowermost mantle. The extent of the regional 
inversion layers varies considerably within our simulations but the location of the thickest anomalous structure is generally centred under the mantle LLVPs. By contrasting $\operatorname{SmKS}$ paths that are expected to completely avoid regional inversion layers with those that should sample the middle of them, it may be possible to test whether the average seismic structure at the top of the core is truly the result of global stratification or if it is instead the signature of large boundary-forced regional inversion layers.

1. Lay, T. \& Young, C. J. The stably-stratified outermost core revisited. Geophysical Research Letters 17, 2001-2004 (1990).

2. Kaneshima, S. Array analyses of SmKS waves and the stratification of Earth's outermost core. Physics of the Earth and Planetary Interiors 276, 234-246 (2018).

3. Helffrich, G. \& Kaneshima, S. Outer-core compositional stratification from observed core wave speed profiles. Nature 468, 807-810 (2010).

4. Buffett, B. Geomagnetic fluctuations reveal stable stratification at the top of the Earth's core. Nature 507, 484-487 (2014).

5. Buffett, B., Knezek, N. \& Holme, R. Evidence for MAC waves at the top of Earth's core and implications for variations in length of day. Geophysical Journal International 204, 17891800 (2016).

6. Buffett, B. A., Mound, J. \& Jackson, A. Inversion of torsional oscillations for the structure and dynamics of Earth's core. Geophysical Journal International 177, 878-890 (2009). 
7. Whaler, K. A. Does the whole of the Earth's core convect? Nature 287, 528-530 (1980).

8. Gubbins, D. Geomagnetic constraints on stratification at the top of Earth's core. Earth, Planets and Space 59, 661-664 (2007).

9. Amit, H. Can downwelling at the top of the Earth's core be detected in the geomagnetic secular variation? Physics of the Earth and Planetary Interiors 229, 110-121 (2014).

10. Lesur, V., Whaler, K. \& Wardinski, I. Are geomagnetic data consistent with stably stratified flow at the core-mantle boundary? Geophysical Journal International 201, 929-946 (2015).

11. Alexandrakis, C. \& Eaton, D. W. Precise seismic-wave velocity atop Earth's core: No evidence for outer-core stratification. Physics of the Earth and Planetary Interiors 180, 59-65 (2010).

12. Irving, J. C. E., Cottaar, S. \& Lekić, V. Seismically determined elastic parameters for Earth's outer core. Science Advances 4, 1-9 (2018).

13. Lister, J. R. \& Buffett, B. A. Stratification of the outer core at the core-mantle boundary. Physics of the Earth and Planetary Interiors 105, 5-19 (1998).

14. Braginsky, S. I. Dynamics of the stably stratified ocean at the top of the core. Physics of the Earth and Planetary Interiors 111, 21-34 (1999).

15. Christensen, U. R. \& Wicht, J. Models of magnetic field generation in partly stable planetary cores: Applications to Mercury and Saturn. Icarus 196, 16-34 (2008).

16. Olson, P., Landeau, M. \& Reynolds, E. Dynamo tests for stratification below the core-mantle boundary. Physics of the Earth and Planetary Interiors 271, 1-18 (2017). 
17. Jones, C. A. Planetary magnetic fields and fluid dynamos. Annual Review of Fluid Mechanics 43, 583-614 (2011).

18. Gubbins, D., Alfè, D., Davies, C. \& Pozzo, M. On core convection and the geodynamo: Effects of high electrical and thermal conductivity. Physics of the Earth and Planetary Interiors 247, 56-64 (2015).

19. Davies, C., Pozzo, M., Gubbins, D. \& Alfè, D. Constraints from material properties on the dynamics and evolution of Earth's core. Nature Geoscience 8, 678-685 (2015).

20. Gubbins, D. \& Davies, C. J. The stratified layer at the core-mantle boundary caused by barodiffusion of oxygen, sulphur and silicon. Physics of the Earth and Planetary Interiors 215, 21-28 (2013).

21. Buffett, B. A. \& Seagle, C. T. Stratification of the top of the core due to chemical interactions with the mantle. Journal of Geophysical Research 115, B04407 (2010).

22. Landeau, M., Olson, P., Deguen, R. \& Hirsh, B. H. Core merging and stratification following giant impact. Nature Geoscience 9, 786-789 (2016).

23. Jacobson, S. A., Rubie, D. C., Hernlund, J., Morbidelli, A. \& Nakajima, M. Formation, stratification, and mixing of the cores of Earth and Venus. Earth and Planetary Science Letters 474, 375-386 (2017).

24. Lister, J. R. Thermal winds forced by inhomogeneous boundary conditions in rotating, stratified, hydromagnetic fluid. Journal of Fluid Mechanics 505, 163-178 (2004). 
25. Nakagawa, T. \& Tackley, P. J. Lateral variations in CMB heat flux and deep mantle seismic velocity caused by a thermal-chemical-phase boundary layer in 3D spherical convection. Earth and Planetary Science Letters 271, 348-358 (2008).

26. Stackhouse, S., Stixrude, L. \& Karki, B. B. First-principles calculations of the lattice thermal conductivity of the lower mantle. Earth and Planetary Science Letters 427, 11-17 (2015).

27. Olson, P., Deguen, R., Rudolph, M. L. \& Zhong, S. Core evolution driven by mantle global circulation. Physics of the Earth and Planetary Interiors 243, 44-55 (2015).

28. Gibbons, S. J., Gubbins, D. \& Zhang, K. Convection in rotating spherical fluid shells with inhomogeneous heat flux at the outer boundary. Geophysical \& Astrophysical Fluid Dynamics 101, 347-370 (2007).

29. Davies, C. J., Gubbins, D. \& Jimack, P. K. Convection in a rapidly rotating spherical shell with an imposed laterally varying thermal boundary condition. Journal of Fluid Mechanics 641, 335-358 (2009).

30. Dietrich, W., Hori, K. \& Wicht, J. Core flows and heat transfer induced by inhomogeneous cooling with sub- and supercritical convection. Physics of the Earth and Planetary Interiors 251, 36-51 (2016).

31. Mound, J. E. \& Davies, C. J. Heat transfer in rapidly rotating convection with heterogeneous thermal boundary conditions. Journal of Fluid Mechanics 828, 601-629 (2017). 
32. Sreenivasan, B. \& Gubbins, D. Dynamos with weakly convecting outer layers: implications for core-mantle boundary interaction. Geophysical \& Astrophysical Fluid Dynamics 102, 395-407 (2008).

33. Sahoo, S., Sreenivasan, B. \& Amit, H. Dynamos driven by weak thermal convection and heterogeneous outer boundary heat flux. Physics of the Earth and Planetary Interiors 250, 35-45 (2016).

34. Olson, P., Landeau, M. \& Reynolds, E. Outer Core Stratification From the High Latitude Structure of the Geomagnetic Field. Frontiers in Earth Science 6, 1-13 (2018).

35. Christensen, U. R. Geodynamo models with a stable layer and heterogeneous heat flow at the top of the core. Geophysical Journal International 215, 1338-1351 (2018).

36. Masters, G., Johnson, S., Laske, G. \& Bolton, H. A shear-velocity model of the mantle. Philosophical Transactions of the Royal Society A: Mathematical, Physical and Engineering Sciences 354, 1385-1411 (1996).

37. Hernlund, J. W. \& McNamara, A. K. The Core-Mantle Boundary Region. In Bercovici, D. (ed.) Mantle Dynamics, 461-519 (Elsevier, Amsterdam, 2015).

38. Gastine, T., Wicht, J. \& Aubert, J. Scaling regimes in spherical shell rotating convection. Journal of Fluid Mechanics 808, 690-732 (2016).

39. Calkins, M. A. et al. The asymptotic equivalence of fixed heat flux and fixed temperature thermal boundary conditions for rapidly rotating convection. Journal of Fluid Mechanics $\mathbf{7 8 4 ,}$ R2 (2015). 
40. Zhang, N. \& Zhong, S. Heat fluxes at the Earth's surface and core-mantle boundary since Pangea formation and their implications for the geomagnetic superchrons. Earth and Planetary Science Letters 306, 205-216 (2011).

41. Glatzmaier, G. A., Coe, R. S., Hongre, L. \& Roberts, P. H. The role of the Earth's mantle in controlling the frequency of geomagnetic reversals. Nature 401, 885-890 (1999).

42. Olson, P., Deguen, R., Hinnov, L. A. \& Zhong, S. Controls on geomagnetic reversals and core evolution by mantle convection in the Phanerozoic. Physics of the Earth and Planetary Interiors 214, 87-103 (2013).

43. Aubert, J., Amit, H., Hulot, G. \& Olson, P. Thermochemical flows couple the Earth's inner core growth to mantle heterogeneity. Nature 454, 758-761 (2008).

44. Gubbins, D., Sreenivasan, B., Mound, J. \& Rost, S. Melting of the Earth's inner core. Nature 473, 361-363 (2011).

45. Ichikawa, H., Tsuchiya, T. \& Tange, Y. The P-V-T equation of state and thermodynamic properties of liquid iron. Journal of Geophysical Research 119, 240-252 (2014).

46. Komabayashi, T. Thermodynamics of melting relations in the system Fe-FeO at high pressure: Implications for oxygen in the Earth's core. Journal of Geophysical Research: Solid Earth 119, 4164-4177 (2014).

47. Brodholt, J. \& Badro, J. Composition of the low seismic velocity E' layer at the top of Earth's core. Geophysical Research Letters 44, 8303-8310 (2017). 
48. Olson, P. \& Christensen, U. R. The time-averaged magnetic field in numerical dynamos with non-uniform boundary heat flow. Geophysical Journal International 151, 809-823 (2002).

49. Aubert, J., Finlay, C. C. \& Fournier, A. Bottom-up control of geomagnetic secular variation by the Earth's inner core. Nature 502, 219-223 (2013).

50. Mound, J., Davies, C. \& Silva, L. Inner core translation and the hemispheric balance of the geomagnetic field. Earth and Planetary Science Letters 424, 148-157 (2015).

51. Finlay, C. C., Olsen, N., Kotsiaros, S., Gillet, N. \& Tøffner-Clausen, L. Recent geomagnetic secular variation from Swarm and ground observatories as estimated in the CHAOS-6 geomagnetic field model. Earth, Planets and Space 68, 112 (2016).

52. Alboussière, T., Deguen, R. \& Melzani, M. Melting-induced stratification above the Earth's inner core due to convective translation. Nature 466, 744-747 (2010).

53. Monnereau, M., Calvet, M., Margerin, L. \& Souriau, A. Lopsided Growth of Earth's Inner Core. Science 328, 1014-1017 (2010).

54. Davies, C. J., Silva, L. \& Mound, J. On the influence of a translating inner core in models of outer core convection. Physics of the Earth and Planetary Interiors 214, 104-114 (2013). 


\begin{tabular}{ccccc} 
Quantity & Definition & Molecular & Turbulent & Simulations \\
\hline Rayleigh & $\widetilde{R a}=\frac{\alpha g_{\mathrm{o}} \beta}{2 \Omega \kappa}$ & $4 \times 10^{13}$ & $2 \times 10^{10}$ & $225-18000$ \\
Ekman & $E=\frac{\nu}{2 \Omega L^{2}}$ & $7 \times 10^{-16}$ & $4 \times 10^{-11}$ & $10^{-6}-10^{-4}$ \\
Prandtl & $\operatorname{Pr}=\frac{\nu}{\kappa}$ & 0.04 & 1 & 1 \\
Reynolds & $R e=U L / \nu$ & $2 \times 10^{9}$ & $4 \times 10^{4}$ & $O\left(10^{1}-10^{3}\right)$ \\
Rossby & $R o=U / 2 \Omega L=R e E$ & $1.5 \times 10^{-6}$ & $1.5 \times 10^{-6}$ & $O\left(10^{-4}-10^{-1}\right)$ \\
\hline
\end{tabular}

Table 1: Nondimensional numbers. 

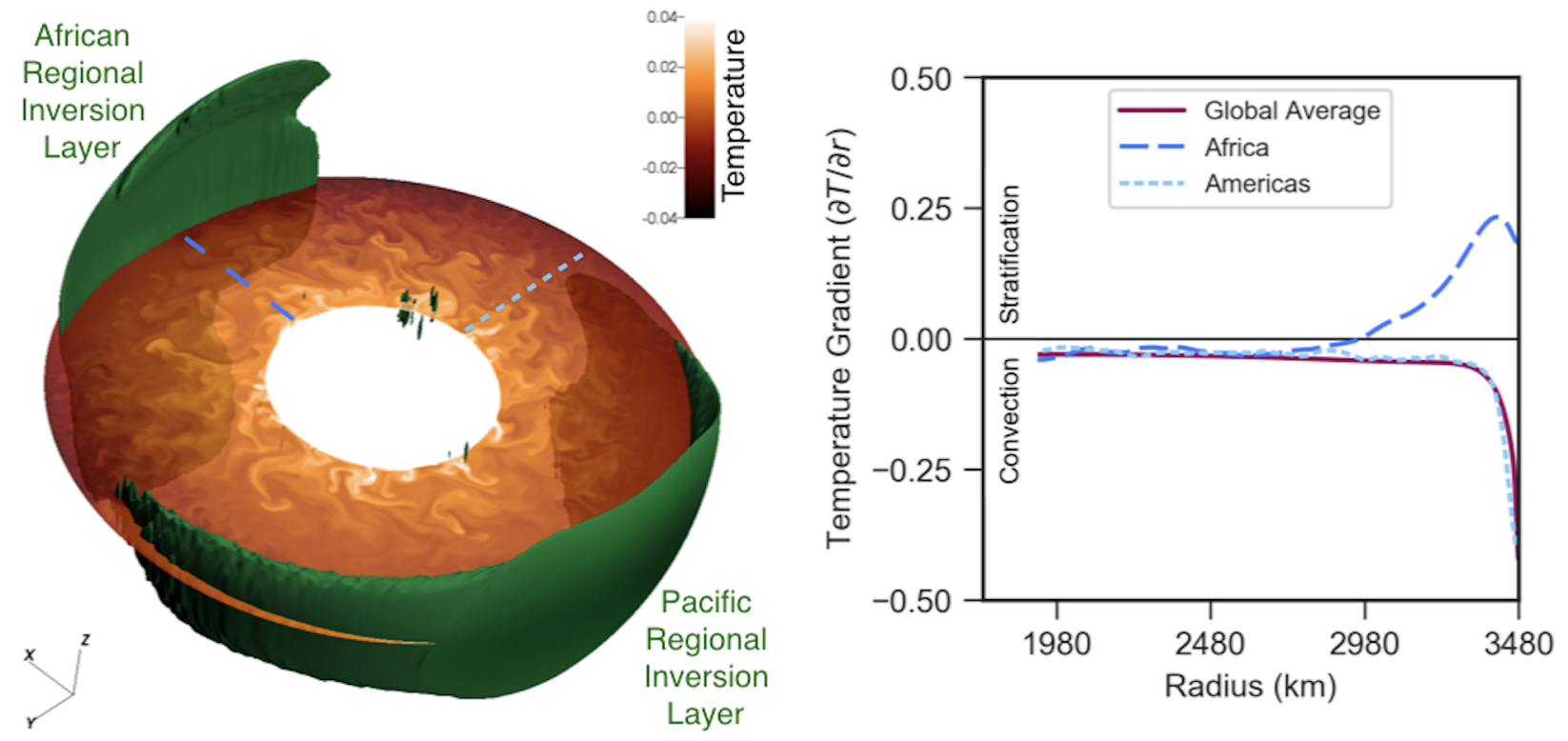

Figure 1: Thermal structure in a simulation with a tomographic pattern of CMB heat flux, $q^{\star}=5.0$, $E=10^{-6}$, and $\widetilde{R a}=1.8 \times 10^{4}$. Left: Green isovolumes denote convectively-stable regions of positive $\partial T / \partial r$ in the time-average; equatorial slice shows the temperature anomaly field at one point in time. Right: Time-averaged profiles of $\partial T / \partial r$ in the top half of the outer core. Regional profiles on the equator $(\theta=\pi / 2)$ are shown for longitudes associated with Africa $(\phi=0$, longdashed blue line) and the Americas ( $\phi=3 \pi / 2$, short-dashed light blue line). The horizontallyaveraged profile is shown by the solid green line. Temperature has been non-dimensionalised as described in the methods section. 



Figure 2: Profiles of time-averaged temperature gradient in the top half of the core. As in figure 1, we consider equatorial profiles under the Americas (left) and Africa (middle), as well as the global average (right). Simulations have a tomographic CMB heat-flux pattern, with $q^{\star}=5.0$, and $E=$ $10^{-4}$ (top), $10^{-5}$ (middle), or $10^{-6}$ (bottom). Colour of the lines indicates the super-criticality of the modified Rayleigh number from 10 times critical (light) to 1000 times critical (dark). Temperature has been non-dimensionalised as described in the methods section. 

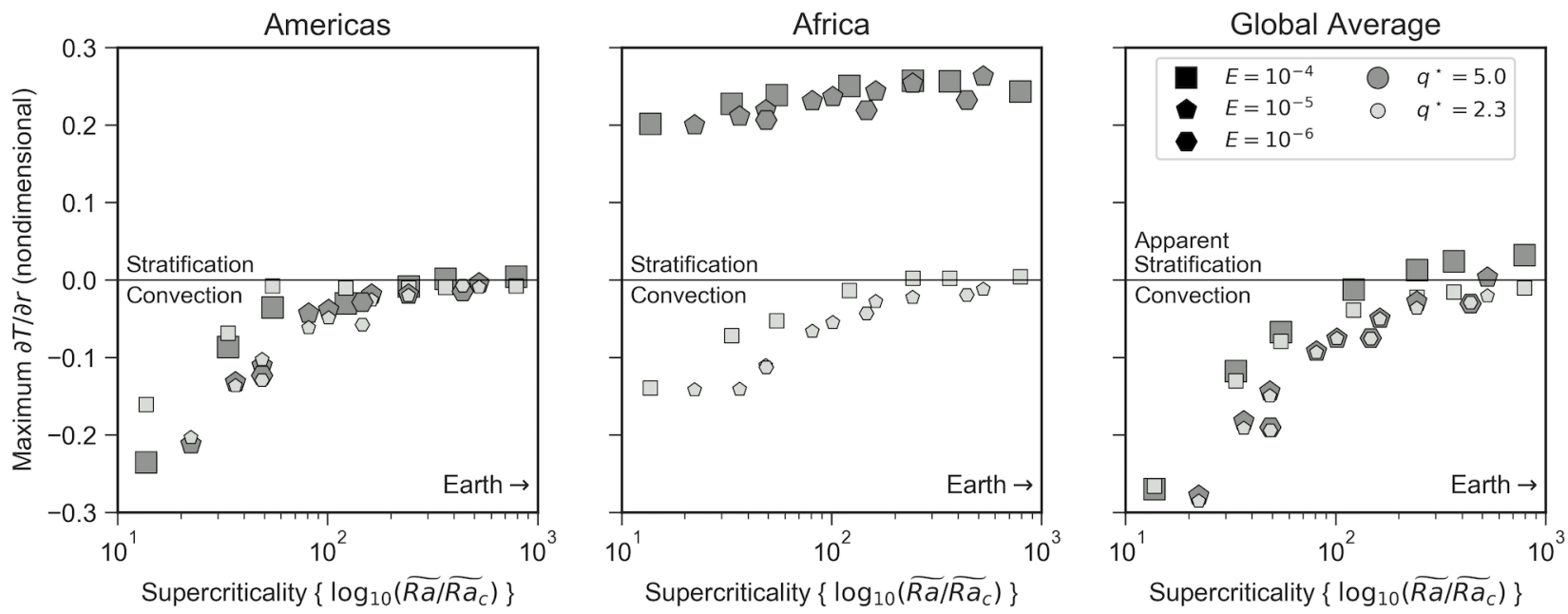

Figure 3: The thermal signature of stratification. The maxima of the profiles of time-averaged temperature gradient (figure 2) are plotted as a function of supercriticality. As supercriticality increases the temperature gradient maxima in our simulations become more positive, corresponding to the formation and strengthening of regional inversion layers and apparent global stratification. Equatorial profiles under the Americas (left) and Africa (middle), and the global average (right). Symbol shape indicates $E=10^{-4}$ (square), $10^{-5}$ (pentagon), or $10^{-6}$ (hexagon). Simulations have a tomographic pattern of CMB heat flux; symbol size and shade indicate $q^{\star}=2.3$ (small, light), or 5.0 (large, dark). 

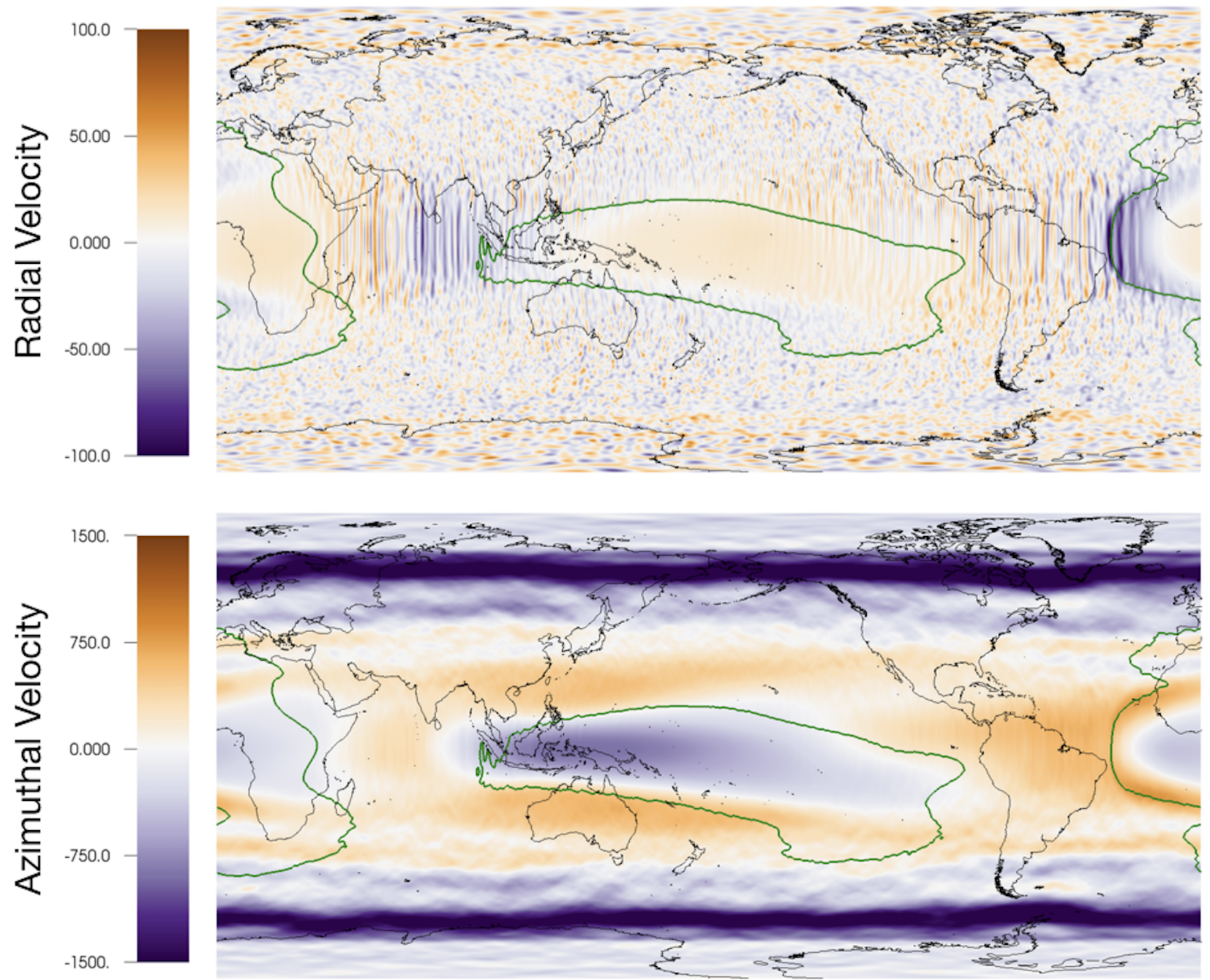

Figure 4: Flow $\sim 100 \mathrm{~km}$ below the CMB for the simulation in figure 1. Time average of the radial velocity (top), azimuthal velocity (bottom), and contours of $\partial T / \partial r=0$ (green). The averaging was done over 37 advection times. The flow velocity is non-dimensionalised as described in the methods section. 


\section{Supplementary Information for "The Apparent Stratifica- tion at the Top of Earth's Liquid Core"}

Jon Mound ${ }^{1}$, Chris Davies ${ }^{1}$, Sebastian Rost ${ }^{1} \&$ Jon Aurnou $^{2}$

${ }^{1}$ School of Earth and Environment, University of Leeds, Leeds LS2 9JT, UK

${ }^{2}$ Department of Earth and Space Sciences, University of California, Los Angeles, California 90095-1567, USA.

Supplemental Movie 1: Equatorial slices (viewed from above, Pacific to left, Africa to right) of thermal structure in the simulation with a tomographic pattern of CMB heat flux presented in figure 1 of the main text. Left: temperature field. Right: radial gradient of temperature. The movie spans approximately 2.6 advection time units, which is approximately $7 \%$ of the total model run.

Supplemental Movie 2: Equatorial slices (viewed from above, Pacific to left, Africa to right) of thermal structure in the simulation with a hemispheric pattern of CMB heat flux presented in supplemental figure 1. Left: temperature field. Right: radial gradient of temperature. The movie spans approximately 2.7 advection time units, which is approximately $7 \%$ of the total model run. 

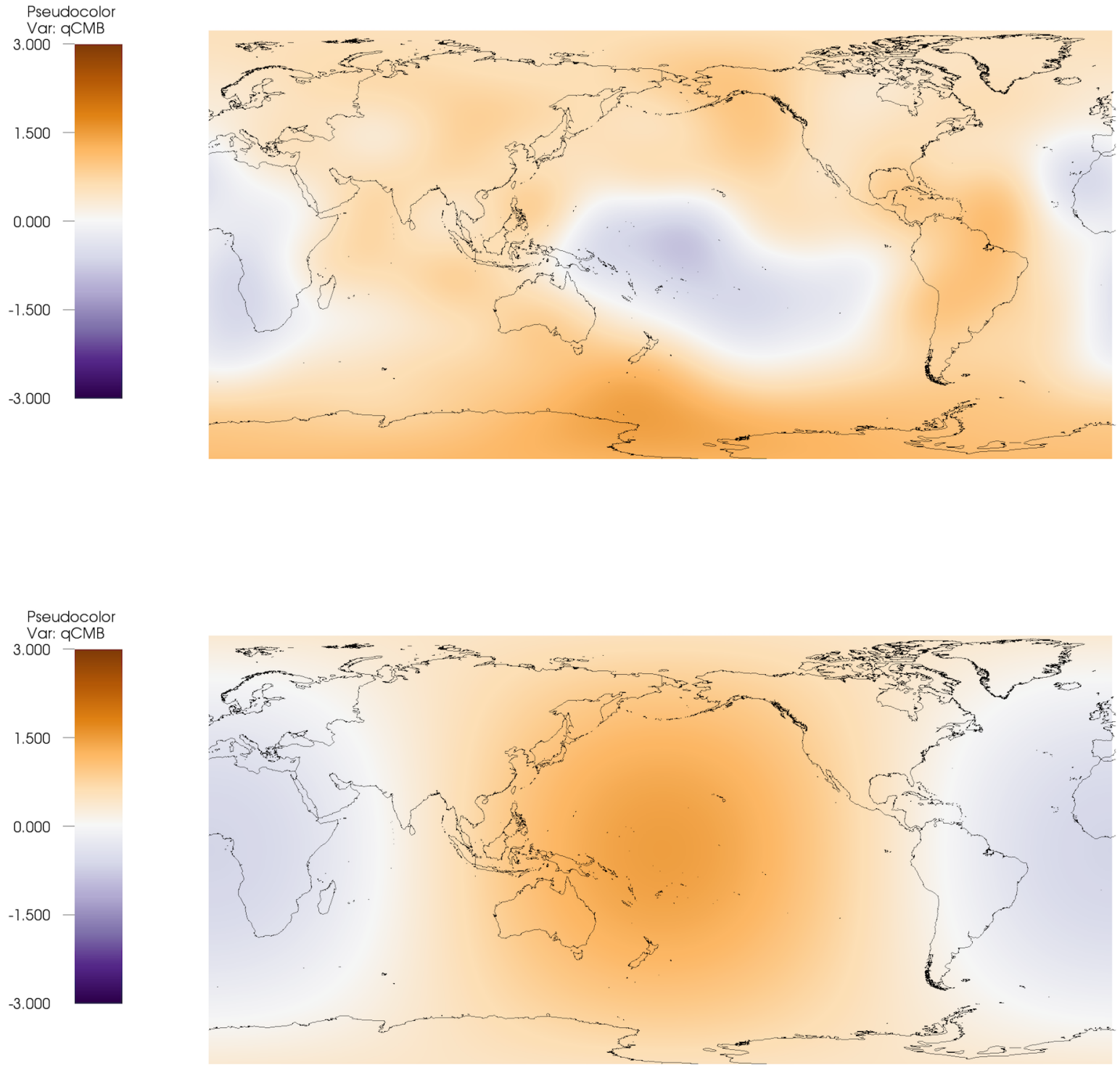

Figure 1: Patterns of CMB heat flux (nondimensional). Upper panel: tomographic pattern. Lower panel: hemispheric pattern. Both cases have $q^{\star}=5.0$. 

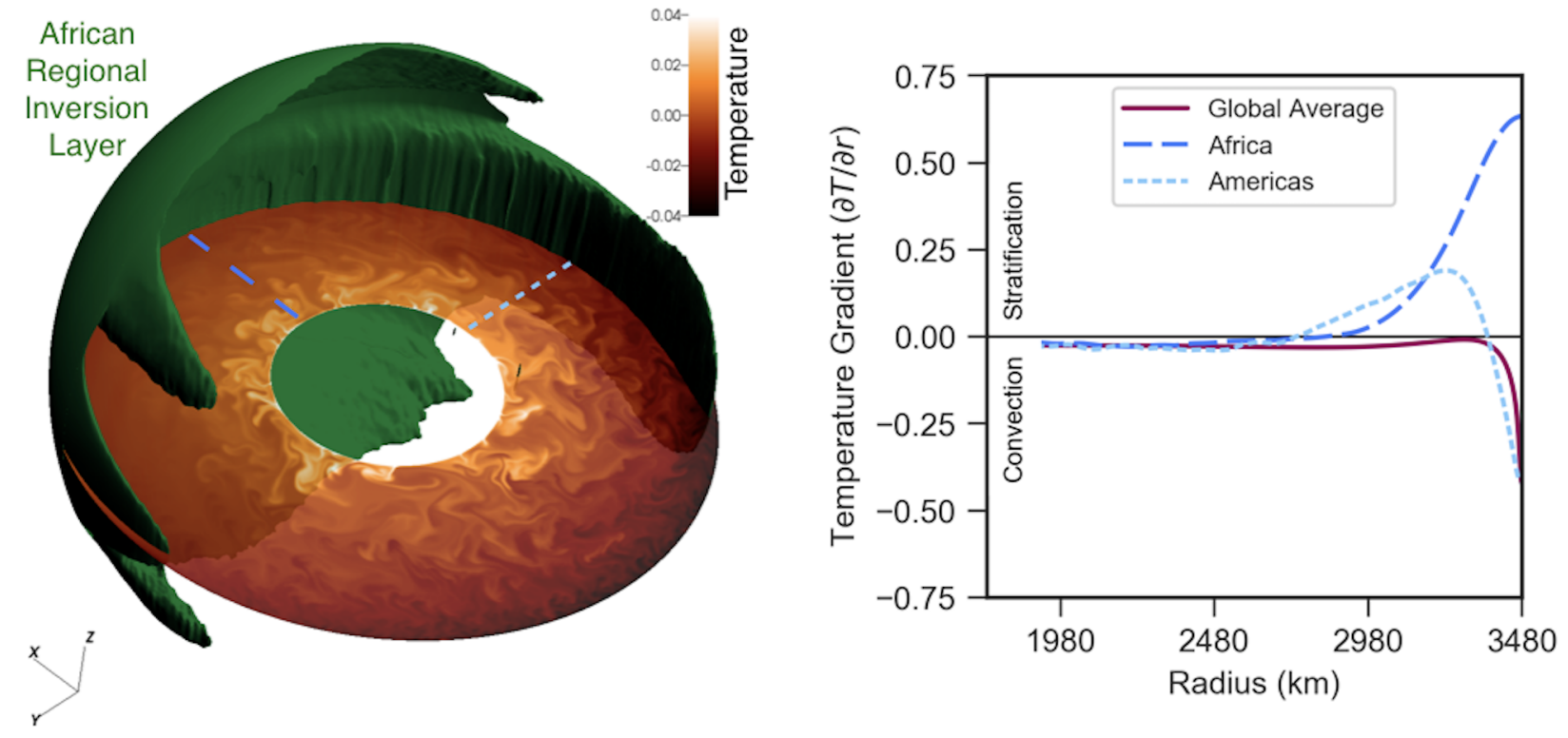

Figure 2: Thermal structure in the simulation with a hemispheric CMB heat flux pattern, $q^{\star}=$ 5.0, $E=10^{-6}$, and $\widetilde{R a}=1.8 \times 10^{4}$. Left: Green isovolumes denote the thermally stratified regional inversion layers $(\partial T / \partial r>0$ in the time-average); equatorial slice shows instantaneous temperature anomalies at one point in time. Right: Time-averaged profiles of temperature gradient $(\partial T / \partial r)$ in the top half of the core $\left(r_{\mathrm{o}} / 2<r<r_{\mathrm{o}}\right)$. Regional profiles on the equator $(\theta=\pi / 2)$ are shown for longitudes associated with Africa $(\phi=0$, long-dash blue line) and the Americas ( $\phi=3 \pi / 2$, short-dash light blue line). The horizontally-averaged profile is shown by the solid magenta line. Temperature has been non-dimensionalised as described in the methods section. 

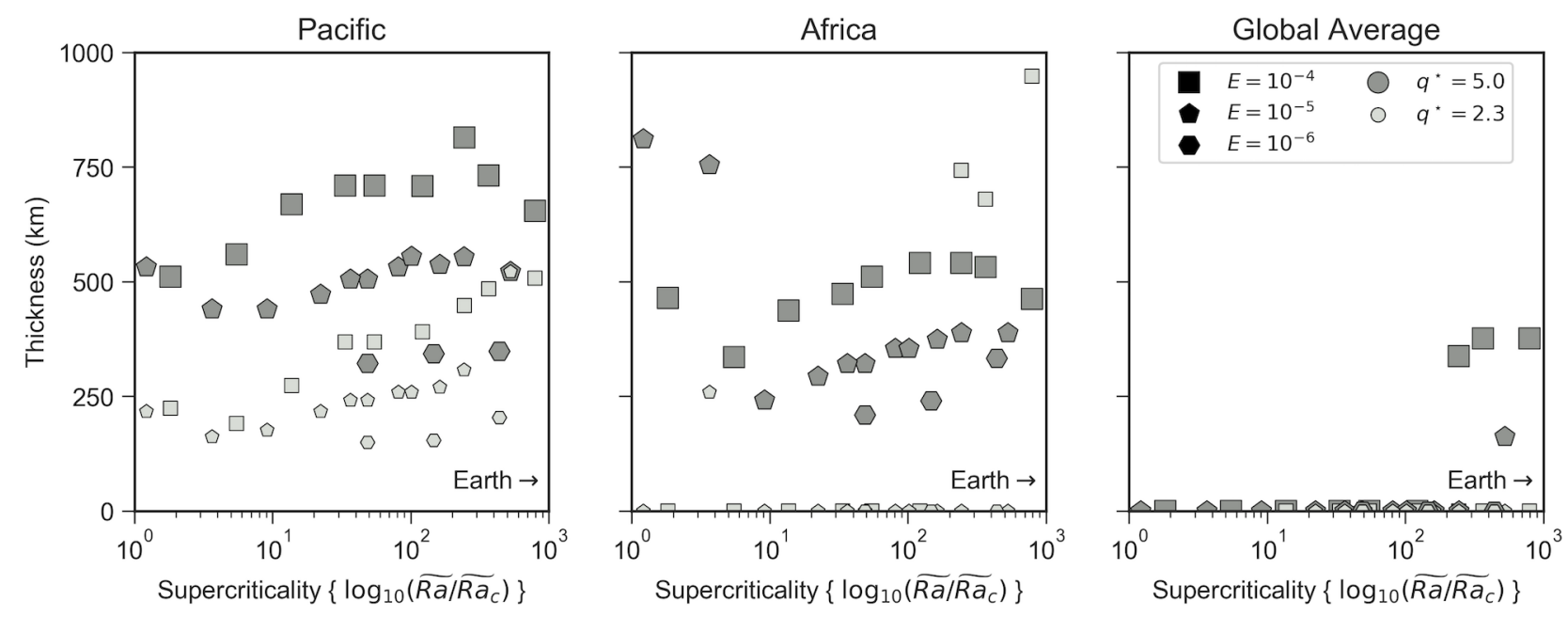

Figure 3: Thickness of the regional inversion layers under the Pacific and Africa, and the thickness of the apparent global stratification, as a function of super-criticality from all simulations with the tomographic CMB heat-flux pattern. Symbol size and colour indicates $q^{\star}=2.3$ (small, light grey), or 5.0 (large, grey). Symbol shape indicates $E=10^{-4}$ (square), $10^{-5}$ (pentagon), or $10^{-6}$ (hexagon). Symbols plotted at zero indicate that there is no regional inversion layer or apparent global stratification for that simulation. 

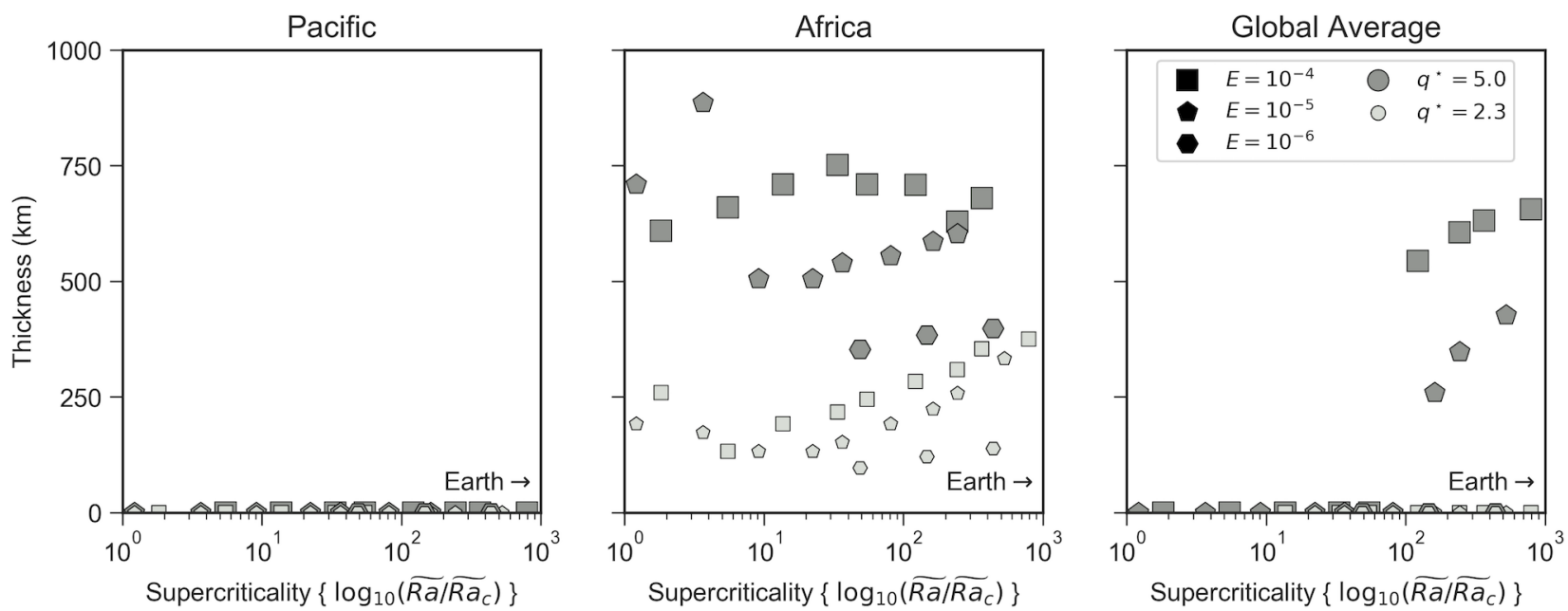

Figure 4: Thickness of the regional inversion layers under the Pacific and Africa, and the thickness of the apparent global stratification, as a function of super-criticality from all simulations with the hemispheric CMB heat-flux pattern (for this pattern no regional inversion layer forms under the Pacific). Symbols plotted at zero indicate that there is no regional inversion layer or apparent global stratification for that simulation. Symbol size and colour indicates $q^{\star}=2.3$ (small, light grey), or 5.0 (large, grey). Symbol shape indicates $E=10^{-4}$ (square), $10^{-5}$ (pentagon), or $10^{-6}$ (hexagon). Symbols plotted at zero indicate that there is no regional inversion layer or apparent global stratification for that simulation. 


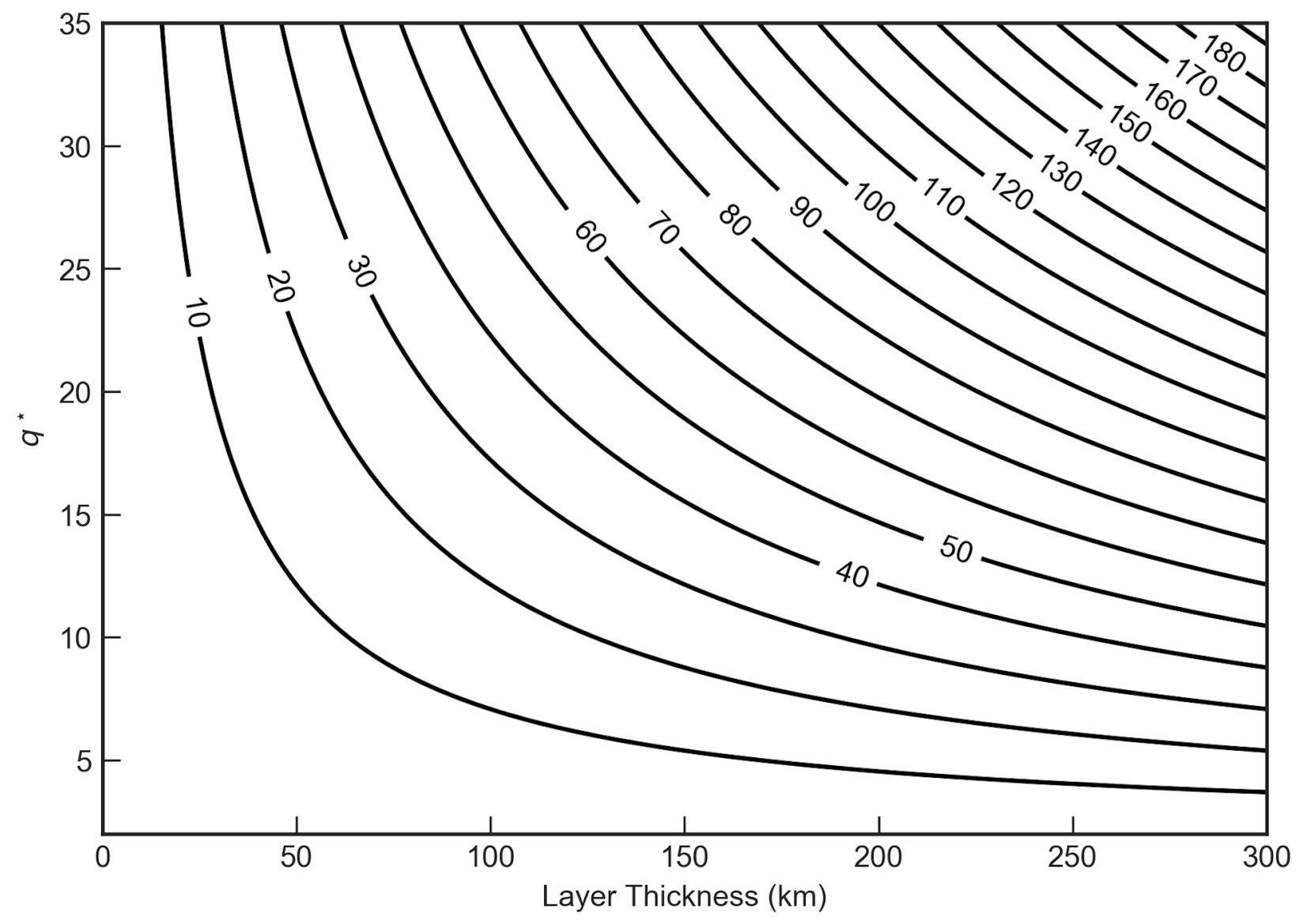

Figure 5: Excess temperature of the stratified regions. Contours of excess temperature (in kelvin) at the top of the core as a function of the layer thickness and the strength of heterogeneity, $q^{\star}$. This example considers a total superadiabatic heat flow across the CMB of $Q_{\text {conv }}=0.6 \mathrm{TW}$, and thermal conductivity $k=100 \mathrm{~W} \mathrm{~m}^{-1} \mathrm{~K}^{-1}$. 


\begin{tabular}{|c|c|c|}
\hline Quantity & Sybol & Value \\
\hline CMB radius & $r_{o}$ & $3.48 \times 10^{6} \mathrm{~m}$ \\
\hline $\mathrm{ICB}$ radius & $r_{i}$ & $1.22 \times 10^{6} \mathrm{~m}$ \\
\hline shell thickness & $L=r_{o}-r_{i}$ & $2.26 \times 10^{6} \mathrm{~m}$ \\
\hline gravitational acceleration at CMB & $g_{o}$ & $10 \mathrm{~m} \mathrm{~s}^{-2}$ \\
\hline thermal expansivity & $\alpha$ & $1.5 \times 10^{-5} \mathrm{~K}^{-1}$ \\
\hline rotation rate & $\Omega$ & $7.29 \times 10^{-5} \mathrm{~s}^{-1}$ \\
\hline thermal diffusivity, molecular & $\kappa_{\mathrm{m}}$ & $1.3 \times 10^{-5} \mathrm{~m}^{2} \mathrm{~s}^{-1}$ \\
\hline thermal diffusivity, turbulent & $\kappa_{\mathrm{t}}$ & $3 \times 10^{-2} \mathrm{~m}^{2} \mathrm{~s}^{-1}$ \\
\hline kinematic viscosity, molecular & $\nu_{\mathrm{m}}$ & $5 \times 10^{-7} \mathrm{~m}^{2} \mathrm{~s}^{-1}$ \\
\hline kinematic viscosity, turbulent & $\nu_{\mathrm{t}}$ & $3 \times 10^{-2} \mathrm{~m}^{2} \mathrm{~s}^{-1}$ \\
\hline CMB superadiabatic heat flow & $Q_{\text {conv }}$ & $0.6 \mathrm{TW}$ \\
\hline thermal conductivity & $k$ & $100 \mathrm{~W} \mathrm{~m}^{-1} \mathrm{~K}^{-1}$ \\
\hline thermal forcing & $\beta=Q_{\mathrm{conv}} /(4 \pi k)$ & $5 \times 10^{8} \mathrm{~K} \mathrm{~m}$ \\
\hline characteristic flow speed & $U$ & $5 \times 10^{-4} \mathrm{~m} \mathrm{~s}^{-1}$ \\
\hline
\end{tabular}

Table 1: Physical parameters. 